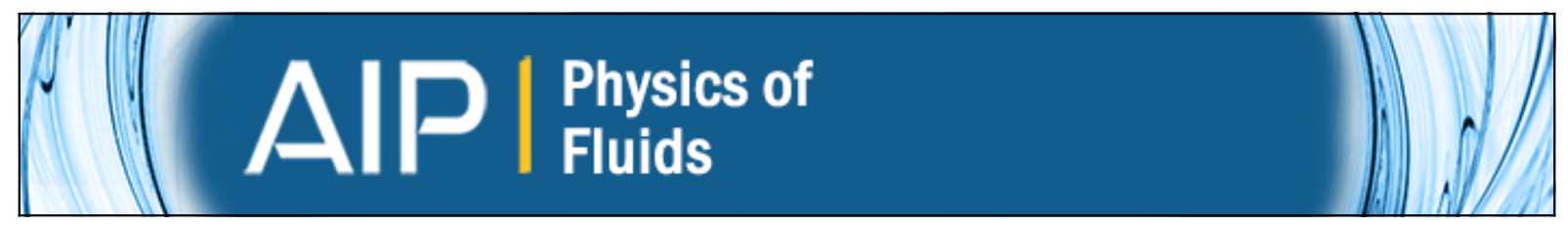

\title{
Impulse and particle dislodgement under turbulent flow conditions
}

Ahmet O. Celik, Panayiotis Diplas, Clinton L. Dancey, and Manousos Valyrakis

Citation: Physics of Fluids (1994-present) 22, 046601 (2010); doi: 10.1063/1.3385433

View online: http://dx.doi.org/10.1063/1.3385433

View Table of Contents: http://scitation.aip.org/content/aip/journal/pof2/22/4?ver=pdfcov

Published by the AIP Publishing

Copyright by the American Institute of Physics. Impulse and particle dislodgement under turbulent flow conditions. Celik, Ahmet 0 . and Diplas, Panayiotis and Dancey, Clinton L. and Valyrakis, Manousos, Physics of Fluids (1994present), 22, 046601 (2010), DOI:http:// dx.doi.org/10.1063/1.3385433

\section{AlP Re-register for Table of Content Alerts}

\section{Create a profile. \\ Sign up today!}




\title{
Impulse and particle dislodgement under turbulent flow conditions
}

\author{
Ahmet O. Celik, ${ }^{1}$ Panayiotis Diplas, ${ }^{1}$ Clinton L. Dancey, ${ }^{2}$ and Manousos Valyrakis ${ }^{1}$ \\ ${ }^{1}$ Department of Civil and Environmental Engineering, Baker Environmental Hydraulics Laboratory, \\ Virginia Tech, Blacksburg, Virginia 24061, USA \\ ${ }^{2}$ Department of Mechanical Engineering, Baker Environmental Hydraulics Laboratory, \\ Virginia Tech, Blacksburg, Virginia 24061, USA
}

(Received 22 December 2009; accepted 11 March 2010; published online 20 April 2010)

\begin{abstract}
In this study, we investigated the role of turbulence fluctuations on the entrainment of a fully exposed grain near threshold flow conditions. Experiments were carried out to measure synchronously the near bed flow velocity and the particle movement for a range of flow conditions and resulting particle entrainment frequencies. We used a simplified bed geometry consisted of spherical particles to reduce the complexities associated with the variations in the bed and flow details in an effort to identify the underlying dominant physical mechanism. An analysis was performed based on common force approximations using near bed flow velocity. Turbulence fluctuations were treated as impulses, which are products of magnitude and duration of applied force. It is demonstrated that besides the magnitude of the instantaneous forces applied on a sediment grain, their duration is important as well in determining whether a particle will be entrained by a turbulent flow event. Frequency of particle entrainment varied remarkably with minute changes in gross flow parameters. Impulse imparted on the sediment grain by turbulent flow was found to be well represented by a log-normal distribution. We obtained a (log-normal) probability density function (pdf) dependent on only the coefficient of variation of the impulse (impulse intensity). Relation of the impulse intensity to the particle Reynolds number, Re*, was established. The sensitivity of the computed impulse to the critical force level, as well as the influence of the critical impulse level on the dislodgement events, was explored. Particle entrainment probabilities were found using the derived pdf as well as experimental observations and a good agreement between the two is reported. Implications of the presented impulse concept and our experimental findings for sediment mobility at low bed shear stress conditions are also discussed. (C) 2010 American Institute of Physics. [doi:10.1063/1.3385433]
\end{abstract}

\section{INTRODUCTION}

The incipient motion condition for granular material defines the stability of erodible beds and constitutes the central problem for sediment transport in rivers, coastal areas, and atmospheric flows. Shields' deterministic framework, ${ }^{1}$ employing time-space average bed shear stress to describe the driving hydrodynamic forces near the bed is the most widely used practical tool and has been for over 70 years. $^{2}$ On the other hand, as a result of decades of work, the literature acknowledges the significance of momentary high turbulent forces on mobilizing the sediment grains at incipient conditions. ${ }^{3-8}$ These forces occur randomly in space and time due to the turbulent flow near the bed. ${ }^{9}$ It is advocated that the movement of a grain begins when the local instantaneous turbulent forces overcome the resisting forces, which are also statistical in nature. ${ }^{8}$ Occasional sediment movement is still possible under turbulent flow conditions where the mean hydrodynamic force is not large enough to entrain the particles. ${ }^{10,11}$ In addition, Paintal, ${ }^{10}$ using long observation periods, showed under flow conditions well below conventional critical conditions, such as those proposed by Shields, ${ }^{1,2}$ that not only the random movements of bed material are observed, but a small increase in the bed mean shear stress causes a significant increase in the movement of bed material. Paintal ${ }^{10}$ reported that at these low stresses the bedload transport rate increases with the 16th power of the boundary shear stress. Helland-Hansen et al. ${ }^{12}$ and Hofland ${ }^{13}$ also made observations under low shear stress and low mobility conditions; those are in qualitative agreement with Paintal's ${ }^{10}$ findings.

Incipient motion criteria utilizing gross flow characteristics, such as those proposed by Shields, do not account for the force fluctuations and therefore are not sufficient to describe the phenomenon at incipient conditions. This view has led many researchers to argue that a unique threshold level in terms of bed shear stress does not exist at which the grain movement suddenly begins. ${ }^{11,14-16}$

Many researchers, in an effort to overcome the limitations of the time-averaged wall shear stress approach, explored the role of turbulent velocity and the resulting fluctuating hydrodynamic forces near the bed on the particle dislodgement, particularly for incipient conditions. Several deterministic and stochastic approaches have been proposed as a result. ${ }^{17-22}$ Common in these approaches is the importance of the magnitude of peaks in the local flow velocity, the streamwise component in particular, and resulting instantaneous forces acting on individual grains in mobilizing these particles. Despite these efforts and partially due to the experimental difficulties in observing the threshold of sediment movement with synchronized flow measurements, which 
also lead to subjectivity in defining the bed mobility, ${ }^{2,17,18}$ the precise determination of the incipient condition remains elusive. Moreover, the nature of the processes that are causing the phenomenon observed by Paintal ${ }^{10}$ has not been explained by the recent methods so far.

The sensitivity of the interactions between local turbulent flow and mobile sediment to even a minute interference, observed during incipient motion experiments, suggests that the investigation of threshold of particle movement requires the use of nonintrusive particle tracking techniques. ${ }^{18,23}$ Balakrishnan, ${ }^{24}$ using a video camera together with local flow velocity measurements, provided evidence contrary to the general consensus, and demonstrated that not all local flow velocity fluctuations well above the mean value result in particle dislodgement under incipient flow conditions. A recent study examined this phenomenon further under well controlled laboratory conditions and demonstrated that it is not the magnitude of the applied force alone that serves as the necessary and sufficient condition for particle entrainment but rather the combination of force and duration, or impulse. ${ }^{25}$ The implication here is that the turbulence induced momentary peak forces acting on the bed material must last long enough to cause entrainment. Although results reported by Diplas et al. ${ }^{25}$ represent the behavior of a test particle in a well controlled environment, this conclusion implies that the impulse potential of a turbulent stream is relevant to the inception of bed material motion, rather than simply the magnitude of the instantaneous forces or timespace-averaged bed shear stress.

The goal of this study is to carry out laboratory flume experiments to further investigate the influence of impulse on entrainment of a single grain for a range of flow strengths and resulting entrainment frequencies. This is pursued by examining long duration data sets of synchronously measured local streamwise flow velocity (and associated impulse values) and the entrainment of a mobile test particle. The former was measured via the use of a laser Doppler velocimeter (LDV), while the latter was monitored using a laserbased particle tracking technique.

Tests were performed in fully developed uniform open channel flow at near threshold conditions. In this study, in an effort to simplify the phenomenon to its most elemental form and facilitate the development of cause and effect relations (while retaining the physics which dominate), the entrainment of an isolated, fully exposed, spherical particle was examined. The preferred mode of particle movement under these conditions is rolling, consistent with observations made in the field for rounded or semirounded particles subjected to near threshold conditions. ${ }^{4,11}$

The impulse concept and the detection of impulses are explained in the following section. The experimental methods are described in detail in Sec. III. Statistics and probability density function (pdf) of impulses detected in various runs are presented in Sec. IV A. We discuss in Secs. IV B and IV $\mathrm{C}$ the sensitivity of the total number of impulses observed and the number of impulses with a potential to yield particle entrainment to the critical force and critical impulse levels, respectively. Following these analyses, the probability of particle entrainment is explored in Sec. IV D. Implications
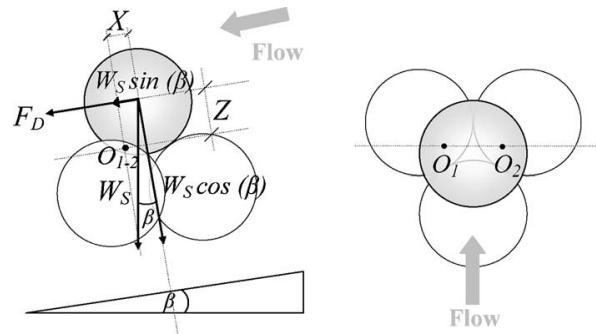

FIG. 1. Definition sketch of the forces acting on a spherical particle resting on identical size densely packed spheres, side view (left) and top view (right) of the bed geometry.

of our findings to sediment movement at low bed shear stresses are discussed in Sec. V. Conclusions are given in Sec. VI.

\section{IMPULSE CONCEPT}

Despite the evidence available in the literature on the dynamical significance of the magnitude of fluctuating local turbulent forces in mobilizing the bed material, the durations over which these turbulent forces act received no attention thus far with the exception of Diplas et al. ${ }^{25}$ They provided evidence that the impulse imparted by near bed turbulent events has to be larger than a critical impulse level to entrain a sediment grain from rest. ${ }^{25}$ Understanding the statistical characteristics of impulse imparted by turbulent flow, rather than just those of the local forces or bed shear stress is essential for describing the incipient bed material motion. In order to detect impulses we used a simple approach in which the force time history is obtained from the local flow velocity, as described in the following section.

\section{A. Impulse detection}

Here, the focus is on a simple setting where the grains are spherical and a mobile particle rests on densely packed identical size particles, as shown in Fig. 1. For the given configuration it is assumed that the forces acting on the mobile particle are submerged particle weight, $W_{S}$, and hydrodynamic force, $F$, assumed to act through the center of gravity of the sphere. ${ }^{26,27}$ Drag force, $F_{D}$, is the prevailing hydrodynamic force component, in line with the flow direction. The lift force, $F_{L}$, is neglected here since drag dominates grain dislodgement for highly exposed particles. ${ }^{28,29}$

Integrating the forcing function, $F(t)$, describing the time history of hydrodynamic force acting upon a particle, between times $t_{1}$ and $t_{2}$, the impulse, related to particle entrainment, is given as

$$
\begin{aligned}
& \text { Impulse }=\int_{t_{1}}^{t_{2}} F(t) d t=\langle F\rangle T \text { with } \\
& F(t) \geq F_{\text {cr }} \text { between } t_{1} \text { and } t_{2},
\end{aligned}
$$

where $T=t_{2}-t_{1}$ is the total duration of the applied force, $\langle F\rangle$ is the time average force over duration $T$, and $F_{\text {cr }}$ is the minimum (critical) force required to initiate bed material motion (obtained from the resting equilibrium conditions ${ }^{18}$ 


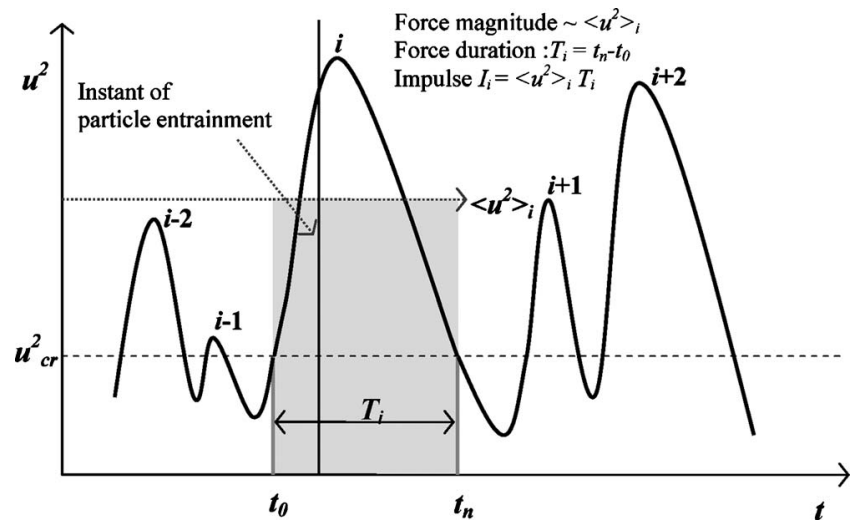

FIG. 2. Representation of the impulse events in the $u^{2}$ time series. The $i$ th event is characterized by $\left\langle u^{2}\right\rangle_{i}$ and $T_{i}$ values, representing force magnitude and duration, the product of which is impulse $=\left\langle u^{2}\right\rangle_{i} T_{i}$ (corresponds to the shaded rectangular area below the $u^{2}$ line). $t_{0}$ and $t_{n}$ were determined by interpolating the adjacent data points in the $u^{2}$ time series. The vertical line between the $t_{0}$ and $t_{n}$ indicates that the particle movement was observed during the $i$ th event.

as discussed later). Since $F_{D}$ is the only hydrodynamic force component considered here, Eq. (1) is treated in the streamwise direction and corresponding $F_{\mathrm{cr}}$, the critical drag force, $F_{D \text { cr }}$, is determined as follows.

$F_{D \text { cr }}$ necessary to first move the spherical grain is obtained by a moment balance about the contact points of the test particle with the base particles. ${ }^{18}$ This is illustrated in Fig. 1 where $O_{1}$ and $O_{2}$ indicate the contact points between the mobile grain and the two downstream base particles. $F_{D \text { cr }}$ can be derived from

$$
F_{D \mathrm{cr}}=f_{V} W_{S}\left[\cos (\beta) \frac{X}{Z}-\sin (\beta)\right],
$$

where the hydrodynamic mass coefficient, ${ }^{18} f_{V}$ $=\left[1+0.5\left(\rho / \rho_{S}-\rho\right)\right], \rho_{S}$ is the density of the particle, $\rho$ is the density of water, $X$ and $Z$ are the lever arms, aligned with and normal to the bed, respectively, and $\beta$ is the angle between the channel bed and the horizontal plane. For steady flows, the relationship between $F_{D}$ and local flow velocity in the streamwise direction, $u$, upstream of the submerged body is given by

$$
F_{D}=\frac{1}{2} \rho u^{2} C_{D} A,
$$

where $C_{D}$ is the drag coefficient and $A$ is the projected grain area perpendicular to flow direction. It is reasonable to estimate the relative level of the instantaneous $F_{D}$ and its temporal variation through the approximation $F_{D}(t) \sim u^{2}(t){ }^{28,30}$ Consequently, the $u^{2}$ time history is utilized in the analysis to estimate the drag force time history. The critical condition for the minimum drag force to initiate motion in terms of flow velocity then can be derived using Eqs. (2) and (3) to yield

$$
u_{\mathrm{cr}}^{2}=\frac{2}{\rho C_{D} A} f_{V} W_{S}\left[\cos (\beta) \frac{X}{Z}-\sin (\beta)\right] .
$$

Here $f_{v}$ is 1.43 for the Teflon ${ }^{\circledR}$ ball that is used in this study and $C_{D}$ is assumed to be $0.9{ }^{28}$ The critical $u^{2}$ value obtained from Eq. (4) is used in our analysis to detect those events in

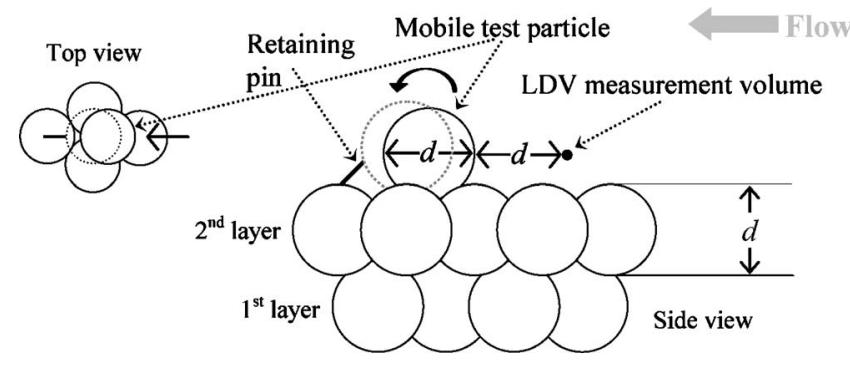

FIG. 3. Side view (right) and top view (upper left corner) sketches of the mobile test particle and pocket geometry (diameter of the grains, $d=12.7 \mathrm{~mm}$ ).

the $u^{2}\left(\sim F_{D}\right)$ time series which exceed the minimum required threshold value $\left(u_{\mathrm{cr}}^{2}\right)$ as illustrated in Fig. 2.

All events with $u^{2} \geq u_{\mathrm{cr}}^{2}$ are detectable within the $u^{2}$ time series together with their times of occurrence and durations, $T_{i}$, (duration over which $u^{2} \geq u_{\mathrm{cr}}^{2}$ ). In addition, time-average $u^{2}$ values, $\left\langle u^{2}\right\rangle_{i}$, can be computed for each such event with $u^{2} \geq u_{\mathrm{cr}}^{2}$, representative of the average drag force, $\left\langle F_{D}\right\rangle_{i}$ of an impulsive event (angle brackets denote averaging over impulse duration). Consequently, relative impulse event magnitudes $I_{i}=\left\langle u^{2}\right\rangle_{i} T_{i}$, hereafter referred to as "impulse," can be obtained. Those events $\left(I_{i}\right)$ associated with particle entrainments can also be identified experimentally by simultaneously measuring the particle movement together with $u(t)$. Accordingly, experiments described in the following section were performed to obtain data sets of local flow velocity and particle entrainment pairs.

\section{EXPERIMENTS}

Incipient motion experiments were conducted in a $20.5 \mathrm{~m}$ long and $0.6 \mathrm{~m}$ wide flume located in the Baker Environmental Hydraulics Laboratory at Virginia Tech. Sporadic entrainment events of a fully exposed, mobile Teflon ${ }^{\circledR}$ spherical particle of diameter, $d=12.7 \mathrm{~mm}$, with a specific gravity of 2.3 , resting on two layers of well packed identical diameter glass spheres, was monitored together with the local flow velocity. The test section was $14 \mathrm{~m}$ downstream from the channel entrance to ensure fully developed turbulent flow conditions. Streamwise component of the local flow velocity, $u$, was measured at one diameter upstream of the test particle along its centerline with a LDV system. Figure 3 illustrates this arrangement. Entrainment of the mobile test particle was recorded utilizing a separate laser-based system that detects its displacement.

Another component of this setup was a retaining pin located $1.5 \mathrm{~mm}$ downstream from the mobile particle (see Fig. 3) to prevent it from being transported downstream from the measurement location. This retaining pin permitted the grain to return to its original position under the action of gravity. This simple but important feature allowed for continuous records of entrainment episodes to be obtained without manual intervention. In this fashion, sets of particle entrainment and local flow velocity data pairs were obtained for various flow conditions. 
TABLE I. Summary of the test conditions for entrainment experiments.

\begin{tabular}{lccccccc}
\hline \hline Run & $\begin{array}{c}U \\
(\mathrm{~m} / \mathrm{s})\end{array}$ & $\begin{array}{c}H \\
(\mathrm{~cm})\end{array}$ & $\mathrm{Re}^{*}$ & $\boldsymbol{\tau}^{*}$ & $\begin{array}{c}f_{E} \\
(\text { Ent./min })\end{array}$ & $\begin{array}{c}u_{\text {mean }} \\
(\mathrm{m} / \mathrm{s})\end{array}$ & $\mathrm{TI}$ \\
\hline E1 & 0.45 & 7.5 & 424 & 0.011 & 6.93 & 0.25 & 0.27 \\
E2 & 0.43 & 8.2 & 413 & 0.010 & 5.73 & 0.24 & 0.27 \\
E3 & 0.43 & 9.0 & 399 & 0.010 & 4.2 & 0.24 & 0.26 \\
E4 & 0.41 & 7.9 & 398 & 0.010 & 2.06 & 0.23 & 0.27 \\
E5 & 0.42 & 8.3 & 385 & 0.009 & 1.33 & 0.23 & 0.27 \\
E6 & 0.40 & 8.6 & 377 & 0.009 & 0.52 & 0.23 & 0.26 \\
E7 & 0.41 & 9.1 & 372 & 0.008 & 0.24 & 0.23 & 0.26 \\
E8 & 0.39 & 8.7 & 364 & 0.008 & 0.14 & 0.22 & 0.27 \\
\hline \hline
\end{tabular}

\section{A. Incipient particle motion detection}

The particle tracking system used in this study employs a single, low power $(25-30 \mathrm{~mW}) \mathrm{He}-\mathrm{Ne}$ laser source and a photodetector, positioned similar to an "electric-eye" arrangement with the voltage output of the photodetector directly related to the position of the mobile grain. The calibration of the He-Ne system was performed in situ using a micrometer and resulted in a resolution of less than $10 \mu \mathrm{m}$ over the $1.5 \mathrm{~mm}$ full streamwise range of particle motion. The LDV and entrainment signals were recorded synchronously via a multichannel signal processor under various flow conditions during the experiments reported here. The sampling frequency in these experiments varied between 250 and $700 \mathrm{~Hz}$. A fraction of the photodetector voltage output from run E1 (see Table I for summary) is shown in Fig. 4 together with simultaneous records of $u^{2}$ and $I_{i}\left(\left\langle u^{2}\right\rangle_{i} T_{i}\right)$. In this example record, both rocking and pivoting of the particle about the contact points are shown. We use the term "pivoting" to indicate those grain movement events which correspond to full grain dislodgement.

The photodetector voltage output was used to identify within the $u^{2}$ and $I_{i}$, time records, the instants when a specific level of particle movement occurred. This is illustrated in Fig. 4. After detecting the instants when the grain moved, a binary $0 / 1$ signal, with " 1 " indicating any detectable particle movement was constructed (Fig. 4). Note that the impulse plot (in the middle) of Fig. 4 includes data for events with $u^{2} \geq u_{\mathrm{cr}}^{2}$ only.

The typical behavior of the test particle during the experiments was observed to be as follows. When the test particle dislodges, it rolls downstream over the valley formed by the pocket arrangement until it reaches the retaining pin (see Fig. 4, instants B and C, respectively). The particle temporarily remains positioned against the pin until the flow induced forces are not strong enough to maintain the position of the particle (Fig. 4, instants C and D, respectively). Then the particle falls back to its original pocket as shown in Fig. 4 between instants D and E. We also observed occasional "rocking" events of the test particle in several runs an example of which is shown in Fig. 4, instant A. At these instants, the particle moves within the pocket but does not reach the retaining pin.

\section{B. Experimental procedure}

We conducted flume experiments under eight different uniform flow conditions that resulted in various (time average) particle entrainment frequencies, $f_{E}$. The following experimental procedure was repeated for each flow condition. First, the experiment was run for sufficiently long time to obtain a stable $f_{E}$ value $(\sim 120 \mathrm{~min})$. Subsequently, under
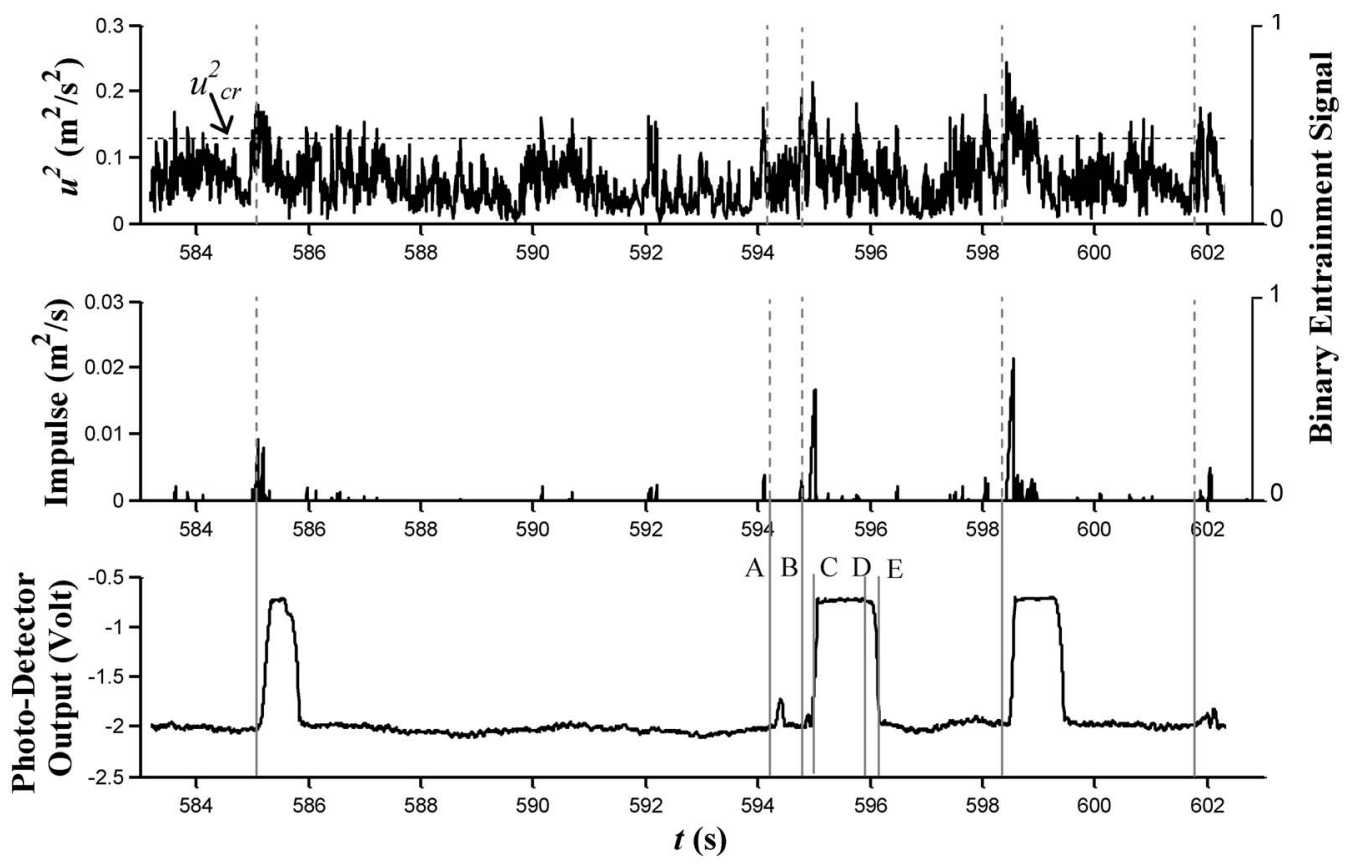

FIG. 4. From top to bottom: representative time series of, $u^{2}$, impulse $\left(\left\langle u^{2}\right\rangle_{i} T_{i}\right)$, and photodetector output, from run E1. Dashed vertical lines in the top two plots indicate detected particle movements. Secondary vertical axes in the top two plots: binary 0/1 signal. Explanation of the solid vertical lines in the bottom plot: (a) beginning of a rocking event, (b) beginning of a pivoting event, (c) instant when the test particle reached the retaining pin, (d) instant when the test particle started rolling back to its original pocket, and (e) instant when the particle reached its original pocket. 
TABLE II. Summary of the impulse parameters obtained from 15 min runs.

\begin{tabular}{lccccc}
\hline \hline & \multicolumn{5}{c}{ Impulse $I=\left\langle u^{2}\right\rangle T$} \\
\cline { 2 - 6 } Run & $\begin{array}{c}I_{\text {mean }} \\
\left(\mathrm{m}^{2} / \mathrm{s}\right) \times 10^{-3}\end{array}$ & $\begin{array}{c}I_{\text {std }} \\
\left(\mathrm{m}^{2} / \mathrm{s}\right) \times 10^{-3}\end{array}$ & $\delta$ & Skewness & Flatness \\
\hline E1 & 2.39 & 2.42 & 1.01 & 3.3 & 14.7 \\
E2 & 2.31 & 2.29 & 0.99 & 3.8 & 11.1 \\
E3 & 2 & 1.9 & 0.95 & 3.5 & 11.4 \\
E4 & 2.1 & 2.07 & 0.97 & 3.6 & 12.6 \\
E5 & 2.11 & 1.96 & 0.93 & 3.8 & 11 \\
E6 & 2.16 & 1.8 & 0.84 & 2.9 & 12.5 \\
E7 & 1.9 & 1.66 & 0.87 & 2.8 & 10.9 \\
E8 & 1.85 & 1.57 & 0.85 & 2.7 & 9 \\
\hline \hline
\end{tabular}

the same flow condition, the local flow velocity and entrainment signals were recorded simultaneously for $15 \mathrm{~min}$. At the completion of the run, velocity profile measurements were obtained one diameter upstream of the test particle but with the test particle removed from its pocket. The bed slope was kept constant at $0.25 \%$ throughout the experiments.

\section{RESULTS AND ANALYSIS}

The analysis reveals that more than $90 \%$ of the particle entrainments in runs E1-E4 and all of the entrainments in the other four runs were associated with events having $u^{2} \geq u_{\mathrm{cr}}^{2}$. This high percentage indicates that our assumption about the dominance of $F_{D}$ on the entrainment of a fully exposed particle is reasonable. It also gives validity to the approach employed here for calculating the $u_{\mathrm{cr}}^{2}$ value, although some uncertainty remains because of the fact that the drag coefficient, $C_{D}$, is not precisely known. ${ }^{28}$

Table I summarizes the flow conditions. It provides the depth average flow velocity, $U$, flow depth, $H$, particle Reynolds number, $\operatorname{Re}^{*}=u^{*} d / \nu$, where $u^{*}$ is the friction velocity, and $\nu$ is the kinematic viscosity ( $u^{*}$ was obtained using the Clauser method $){ }^{31}$ shields stress, $\tau^{*}=\tau_{0} /\left(\rho_{s}-\rho\right) g d$, where $\tau_{0}$ is time-average bed shear stress, $g$ is gravitational acceleration; $f_{E} ; u_{\text {mean }}$ which is the time average value of $u$ at a point one diameter upstream of the test particle along its
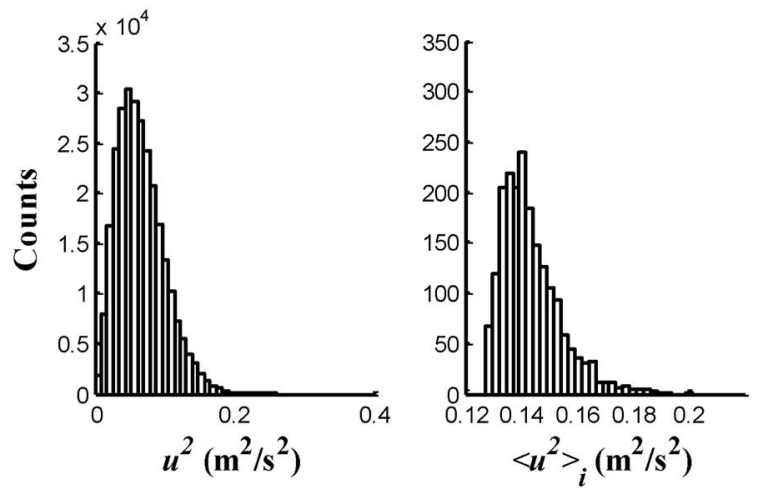

center, and turbulence intensity given by $u_{\text {rms }}^{\prime} / u_{\text {mean }}$, where $u_{\mathrm{rms}}^{\prime}$ is the root-mean-square of the turbulent velocity fluctuations, $u^{\prime}$.

Significant variation in the frequency of grain entrainment occurs with only minute changes in the gross flow parameters. Table I shows that a $14 \%$ increase in $\operatorname{Re}^{*}(\sim 35 \%$ increase in bed shear stress, $\left.\tau_{0} \sim u^{*^{2}}\right)$ is accompanied by a nearly 50-fold increase in $f_{E}$. This result is in qualitative agreement with the findings of Paintal ${ }^{10}$ and Helland-Hansen et al., ${ }^{12}$ and consistent with more limited flume observations of Hofland. ${ }^{13}$ It is also noted that the variation in the turbulence intensity measured in the immediate vicinity of the particle for all eight experiments is almost negligible. These findings exemplify the inadequacies of incipient motion models that employ time-space average flow parameters and suggest that deterministic and stochastic models that depend on local turbulence intensity to define the threshold of particle movement must be used cautiously.

Events, for which $u^{2} \geq u_{\mathrm{cr}}^{2}$, were obtained for all runs (E1-E8) by performing the analysis described in Sec II A via an impulse detection code. The statistics of detected impulse events are presented in Table II for E1-E8. The histograms of $u^{2} ;\left\langle u^{2}\right\rangle_{i}$ representing drag force averaged over the impulse duration for which $u^{2} \geq u_{\mathrm{cr}}^{2} ; T_{i}$ which is the impulse duration and $I_{i}$, impulse from run E1 are given in Fig. 5. A number of pdfs has been proposed for $u^{2}$ in studies concerning the sediment entrainment. ${ }^{18,19,21}$ Based on the assumption that the drag force is proportional to $u^{2}$, in combination with a Gaussian distribution for $u$, Papanicolaou ${ }^{18}$ proposed a chisquared distribution to describe instantaneous drag force, which was later modified by Hofland and Battjes ${ }^{30}$ to account for the relative turbulence intensity. But the distribution of the impulse, impulse duration or force magnitude associated with impulse events, to the writers' knowledge, has never been examined in sediment transport research. The histogram of impulse is positively skewed with a long tail as shown in Fig. 5. These distribution characteristics are due to very high magnitude but rare impulse events. The rare impulse events, described by relatively long durations and a range of $\left\langle u^{2}\right\rangle_{i}$ values are in fact those responsible for particle movement (see Sec. IV B). It is important to note that the duration of impulse events $\left(T_{i}\right)$ shows more than an order-of-

FIG. 5. Histograms of $u^{2},\left\langle u^{2}\right\rangle_{i}, T_{i}$, and $I_{i}$ from left to right for the run E1. Nearly 280000 data points (counts) for $u^{2}$ and total of 1978 data points for $\left\langle u^{2}\right\rangle_{i}$, $T_{i}$, and $I_{i}$ are represented in each histogram. 


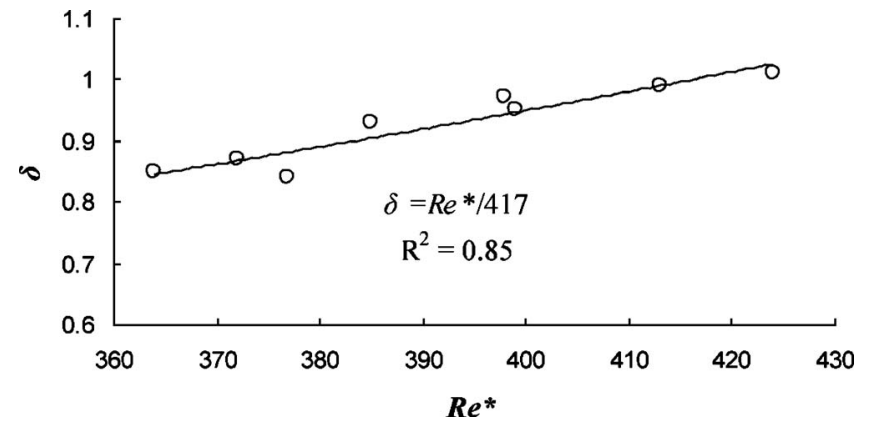

FIG. 6. Relationship between impulse intensity, $\delta$, and particle Reynolds number, $\mathrm{Re}^{*}$.

magnitude variation but a relatively narrow range of $\left\langle u^{2}\right\rangle_{i}\left(0.13-0.2 \mathrm{~m}^{2} / \mathrm{s}^{2}\right)$, in all runs (Fig. 5). The variability of event durations is much more pronounced compared to force magnitudes and thus the durations, to a greater extent, influence the impulse values.

\section{A. Distribution of impulse}

The impulse statistics and histograms indicate that the impulse distribution is heavily skewed. A suitable function that describes data with such properties is the log-normal distribution function. Cheng and $\mathrm{Law}^{32}$ proposed a lognormal distribution to describe the bed shear stress fluctuations. Another example of the use of log-normal distribution function in sediment research is provided by Wu et al. ${ }^{33}$ Recently, Mouri et $a l .{ }^{34}$ reported log normality in energy fluctuations obtained from velocity measurements for a wide range of turbulence scales at various flow conditions. It is generally argued that the origin of log normality lies in the multiplicative stochastic processes, i.e., as a result of independent stochastic variables inherent to turbulent flow phenomena. ${ }^{34,35}$ Impulse, the product of turbulent forces and their durations near the bed may be described by a log-normal distribution. If the impulse is nondimensionalized with its ensemble average value, $\hat{I}=I / I_{\text {mean }}$, then the lognormal probability density function for the dimensionless impulse, $\hat{I}$ as a function of the impulse intensity, $\delta=I_{\text {std }} / I_{\text {mean }}$ is given by

$$
f(\hat{I})=\frac{1}{\sqrt{2 \pi \ln \left(1+\delta^{2}\right)} \hat{I}} \exp \left[-\frac{\left(\ln \hat{I}+\ln \sqrt{1+\delta^{2}}\right)^{2}}{2 \ln \left(1+\delta^{2}\right)}\right] .
$$

The derivation of Eq. (5) is given in the Appendix. Note that $\delta=\delta_{I}$, the latter being the intensity of the dimensionless impulse, $\hat{I}$. Table II gives the calculated $\delta$ values for all eight runs. A linear relationship exists between $\delta$ and $\operatorname{Re}^{*}$ for the limited range of uniform flow conditions tested here (Fig. 6). A linear regression approach provides the following expression:

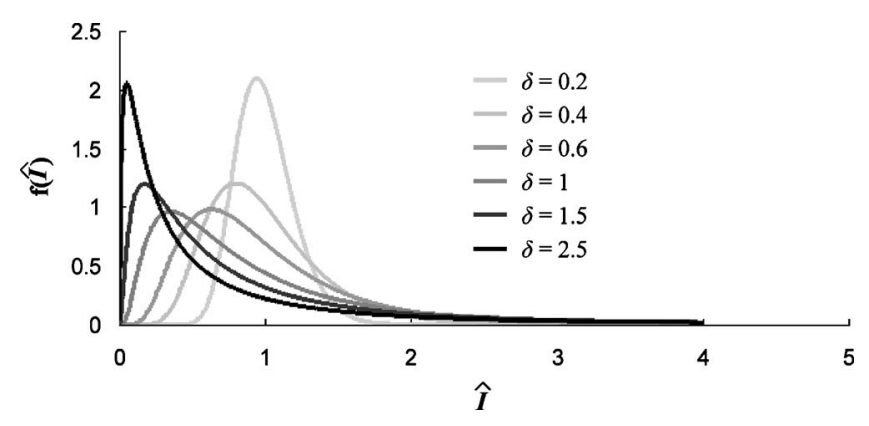

FIG. 7. Plots of the function given by Eq. (5) for a range of $\delta$ values.

$$
\delta=\frac{\mathrm{Re}^{*}}{417},
$$

with a coefficient of determination of 0.85 .

Figure 7 shows the influence of $\delta$ on the distribution of $\hat{I}$ [Eq. (5)]. Equation (5) approaches a normal distribution for $\delta<0.2$. For higher $\delta$ values, on the other hand, the distribution becomes positively skewed. For all $\delta$ values that are of interest here (Table II) and shown in Fig. 7, the difference in the right tails of pdfs is clear and significant. These deviations in the shape of the tail with small variations in $\delta$ essentially dictate the probability of occurrence of extreme impulse events above a high (critical) level. As it will be discussed in the next section, critical impulse level ${ }^{25}$ itself, which has different values in dimensionless form for each run, is also crucial in determining the probability of occurrence of impulse events with a potential to entrain the particle.

Measured pdfs of impulses, obtained from normalized histograms, are compared with Eq. (5) in Fig. 8 for all eight runs. $\delta$ values were determined from the data for each particular run and were used in Eq. (5). It can be seen that the derived pdf describes the data well. In the construction of the pdfs shown in Fig. 8, the tail of the distribution for high impulse is emphasized. This tail characterizes the extreme impulse events and hence it is most relevant to particle entrainment for near threshold conditions, as discussed later in Sec. IV D. The selection of the optimum number of bins $(\sim 25)$ used to resolve the tail necessarily results in poor resolution at the other extreme of the distribution (the rising leg and peak). In order to show the tail sections better, a semilogarithmic plot of Eq. (5), together with all the experimental data, is presented in Fig. 9 covering the range of test conditions ( $\delta=0.7$ and 1.1). Despite the scatter of data, especially at higher $\hat{I}$ values $(\hat{I}>3)$, Eq. (5) predicts the overall trend well. Since the rising leg and the peak in the pdfs are now involved in rest of the analysis, no uncertainty due to the differences/lack of data points in this section of the pdf was introduced. Therefore no attempts, such as employing arbitrary $\delta$ values for different sections of the pdf, have been made. 

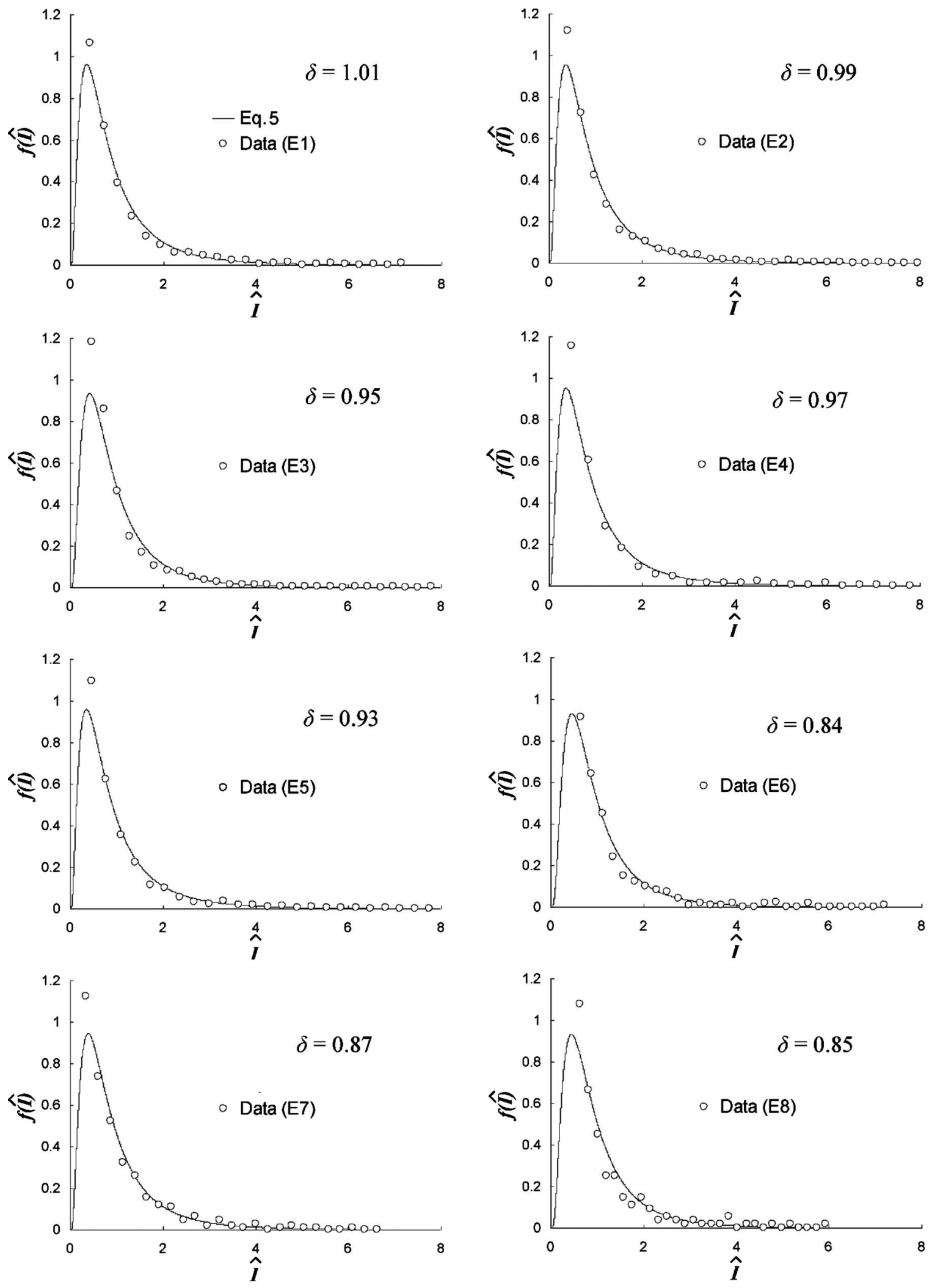

FIG. 8. Comparison of Eq. (5) with measured pdfs for E1-E8. Solid lines are used to show pdfs obtained from Eq. (5).

Furthermore, low amplitude impulse events (where $\hat{I}<1)$ in the rising legs of the pdfs are characterized by combinations of very short $T_{i}$ and very high $\left\langle u^{2}\right\rangle_{i}$ values, or vice versa. Close inspection of the data reveals that the former may not be detected accurately even at turbulent resolving sampling frequencies (i.e., peaks occurring within less than $5 \mathrm{~ms}$ ). The latter are, on the other hand, extremely rare. Given that the analysis is sensitive to the features of detected 


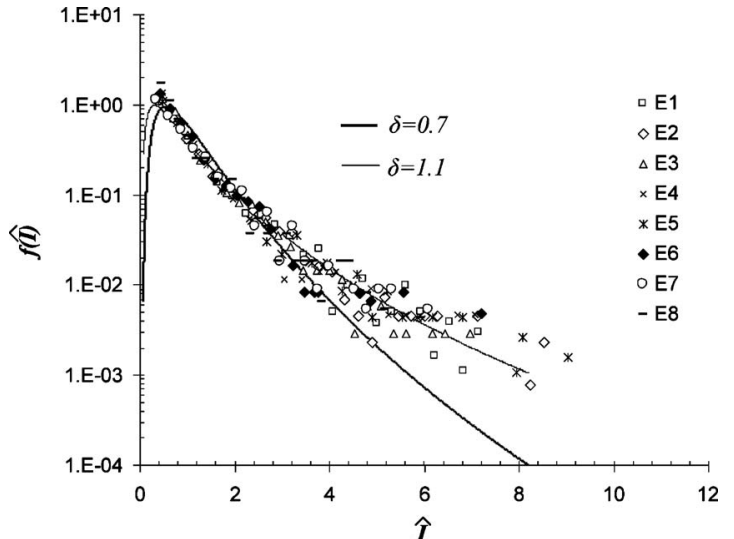

FIG. 9. Semilogarithmic plot of measured pdfs from all eight runs. Equation (5) is also presented with $\delta=0.7$ and 1.1 for comparison.

impulses, the following Secs. IV B and IV C are presented to examine the role of critical $u^{2}$ level $\left(u_{\mathrm{cr}}^{2}\right)$ on the number and statistics of the detected impulses.

\section{B. Critical $u^{2}$ and number of impulses}

Values of $\left\langle u^{2}\right\rangle_{i}$ are plotted with respect to corresponding $T_{i}$ in Fig. 10 for all impulse events and including the events associated with full particle dislodgement (the latter are shown in the figure with solid circles) for runs E1 and E4. Plots given in Fig. 10 clearly demonstrate that, in agreement with the findings of Diplas et al. ${ }^{25}$ although $F_{D} \geq F_{D \text { cr }}$ is necessary for the initiation of motion, this condition alone is not sufficient to predict full grain dislodgement. Contrary to common force-balance models and other stochastic approaches, only particular combinations of $\left\langle u^{2}\right\rangle_{i}$ and $T_{i}\left(u^{2} \geq u_{\mathrm{cr}}^{2}\right)$ with moderate to large durations, yielded grain dislodgement. In fact, the most recent incipient motion models ${ }^{16,19,20}$ would significantly overestimate the particle entrainment rate under similar flow and bed conditions. This is true since all of the detected events with $u^{2} \geq u_{\mathrm{cr}}^{2}$, for instance all 1978 events in run E1, are routinely expected to entrain the particle in such mechanistic models because these events would cause very high instantaneous drag forces on the particle. On the contrary, our experiment shows that less than $7 \%$ of these events caused particle entrainment in run E1. This finding is consistent with Balakrishnan's ${ }^{24}$ observations. The total number of impulse events, $n_{T}$, for which $u^{2} \geq u_{\mathrm{cr}}^{2}$, and the number of impulse events $\left(u^{2} \geq u_{\mathrm{cr}}^{2}\right)$ that yield particle dislodgement, $n_{E}$, both observed over a $15 \mathrm{~min}$ sampling period, are given for all runs in Table III. Note that
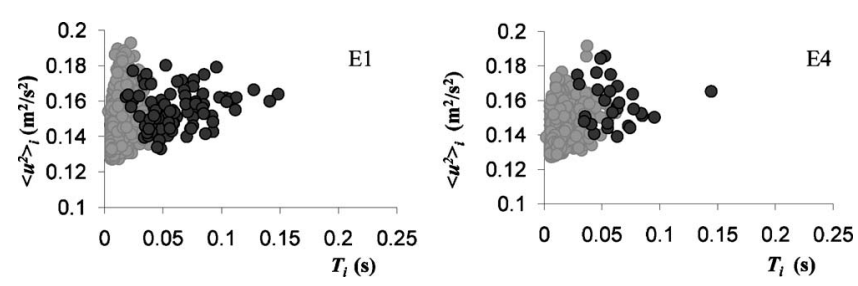

FIG. 10. $\left\langle u^{2}\right\rangle_{i}$ vs $T_{i}$ plots. 1978 data points from run E1 (left), 1101 data points from run E4 (right). Black circles indicate $\left\langle u^{2}\right\rangle_{i}, T_{i}$ combinations that are associated with full particle dislodgement (pivoting).
TABLE III. Number of impulse events and particle movements observed for 15 min. Note that $n_{E}=n_{P}+n_{R}$.

\begin{tabular}{lccc}
\hline \hline & & \multicolumn{2}{c}{ No. of particle movements $n_{E}$} \\
\cline { 3 - 4 } Run & No. of impulse events $n_{T}$ & Pivoting $n_{P}$ & Rocking $n_{R}$ \\
\hline E1 & 1978 & 104 & 29 \\
E2 & 1554 & 86 & 17 \\
E3 & 1262 & 63 & 16 \\
E4 & 1101 & 31 & 15 \\
E5 & 722 & 20 & 8 \\
E6 & 416 & 8 & 4 \\
E7 & 362 & 4 & 1 \\
E8 & 249 & 1 & 3 \\
\hline
\end{tabular}

$n_{E}=n_{P}+n_{R}$, where $n_{P}$ and $n_{R}$ are the number of pivoting and rocking events, respectively. As shown in Table III, the difference between the $n_{T}$ and $n_{E}$ values is dramatic for all runs. Overall, less than an-order-of magnitude increase in $n_{T}$ corresponds to two-orders-of-magnitude increase in the $n_{E}$. This observation supports the idea that the force magnitude alone is not sufficient to describe the particle entrainment.

In order to investigate the origins of this remarkable variability in the number of impulses with minute changes in the flow conditions, the role of critical $u^{2}\left(u_{\mathrm{cr}}^{2}\right)$ level on the number and statistics of the detected impulses was examined. This was done artificially, not by running additional experiments with different grain density or size, but rather by adjusting the level of $u_{\mathrm{cr}}^{2}$ in the analysis of the current series of experiments. This approach is comparable to that by varying the gross flow parameters slightly as was the procedure in the actual experiments. In this analysis, data from run E1 were used. Eight arbitrarily chosen critical $u^{2}$ values slightly above and below the original critical level $\left(u_{\mathrm{cr}}^{2} \pm \Delta u^{2}\right)$, as illustrated in Fig. 11(a), were selected to identify impulse events, now associated with drag force above the new critical level. Table IV gives the critical $u^{2}$ value that is used; the resulting number of detected impulses, mean and standard deviations of $\left\langle u^{2}\right\rangle_{i}, T_{i}$, and impulse, respectively, and the impulse intensity. The row with bold font in Table IV indicates the results from the conditions where the original critical $u^{2}$ obtained from Eq. (4) was used to detect the impulses.

It is observed that the number of detected impulses is very sensitive to the chosen critical level of $u^{2}$. Slightly shift-
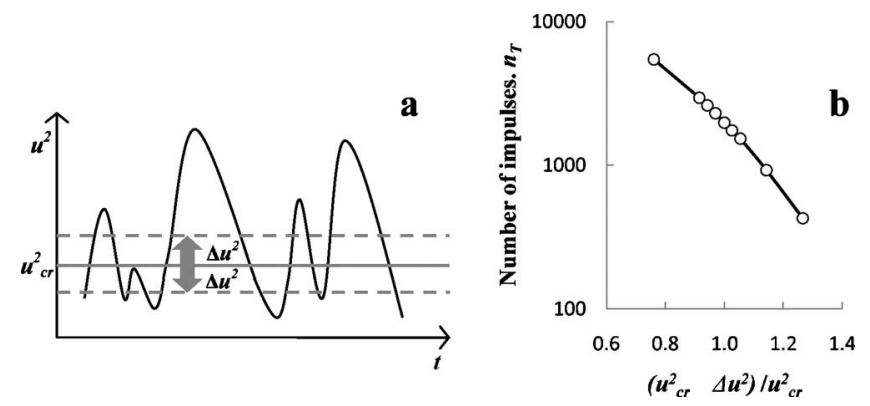

FIG. 11. (a) Illustration of the approach used for varying the critical $u^{2}$. (b) Number of detected impulses vs the ratio of critical $u^{2}$ level used to the original $u_{\mathrm{cr}}^{2}$. 
TABLE IV. Summary of the results from conditions where various $u^{2}$ critical values were used for run E1.

\begin{tabular}{ccccccccc}
\hline \hline $\begin{array}{c}\text { Critical } u^{2} \\
\left(\mathrm{~m}^{2} / \mathrm{s}^{2}\right)\end{array}$ & $\begin{array}{c}\text { Number } \\
\text { of detected impulses } \\
\left(n_{T}\right)\end{array}$ & $\begin{array}{c}\left\langle u^{2}\right\rangle_{i \text { mean }} \\
\left(\mathrm{m}^{2} / \mathrm{s}^{2}\right)\end{array}$ & $\begin{array}{c}\left\langle u^{2}\right\rangle_{i \text { std }} \\
\left(\mathrm{m}^{2} / \mathrm{s}^{2}\right)\end{array}$ & $\begin{array}{c}T_{i \text { mean }} \\
(\mathrm{s})\end{array}$ & $\begin{array}{c}T_{i} \text { std } \\
(\mathrm{s})\end{array}$ & $\begin{array}{c}I_{i} \text { mean } \\
\left(\mathrm{m}^{2} / \mathrm{s}\right)\end{array}$ & $\begin{array}{c}I_{i} \text { std } \\
\left(\mathrm{m}^{2} / \mathrm{s}\right)\end{array}$ & $\delta$ \\
\hline 0.160 & 427 & 0.176 & 0.010 & 0.013 & 0.008 & 0.0022 & 0.0016 & 0.72 \\
0.144 & 925 & 0.161 & 0.010 & 0.014 & 0.011 & 0.0023 & 0.0019 & 0.83 \\
0.133 & 1532 & 0.150 & 0.010 & 0.015 & 0.013 & 0.0023 & 0.0022 & 0.96 \\
0.130 & 1748 & 0.147 & 0.010 & 0.016 & 0.014 & 0.0024 & 0.0023 & 0.96 \\
$\mathbf{0 . 1 2 6}$ & $\mathbf{1 9 7 8}$ & $\mathbf{0 . 1 4 3}$ & $\mathbf{0 . 0 1 1}$ & $\mathbf{0 . 0 1 6}$ & $\mathbf{0 . 0 1 5}$ & $\mathbf{0 . 0 0 2 4}$ & $\mathbf{0 . 0 0 2 4}$ & $\mathbf{1 . 0 1}$ \\
0.123 & 2302 & 0.140 & 0.011 & 0.017 & 0.016 & 0.0024 & 0.0025 & 1.04 \\
0.119 & 2615 & 0.136 & 0.011 & 0.017 & 0.016 & 0.0024 & 0.0026 & 1.08 \\
0.116 & 2948 & 0.133 & 0.011 & 0.017 & 0.017 & 0.0024 & 0.0026 & 1.08 \\
0.096 & 5456 & 0.114 & 0.012 & 0.021 & 0.023 & 0.0025 & 0.0032 & 1.28 \\
\hline \hline
\end{tabular}

ing the critical $u^{2}$ level up or down results in a significant change in the number of detected impulses. The number of impulses increases by an order of magnitude when the critical $u^{2}$ level is shifted downward by only about $40 \%$ as shown in Fig. 11(b). The latter also corresponds to a hypothetical $25 \%$ decrease in the particles density [Eq. (4)]. This is due to the fact that as the critical $u^{2}$ is reduced, those events that were initially below $u_{\mathrm{cr}}^{2}$ now become important (i.e., detectable). The significant increase in the number of impulses in this analysis, similar to what was observed in the original experiments, suggests that the temporal features, resulting from the underlying structure of turbulence, together with the critical $u^{2}$ level, plays a crucial role in the significant variation of number of impulse events observed here.

The $\left\langle u^{2}\right\rangle_{i \text { mean }}$ as well as $T_{i \text { std }}$ increase linearly with increasing critical $u^{2}$ level while the $\left\langle u^{2}\right\rangle_{i \text { std }}$ and $T_{i \text { mean }}$ decrease. The change in $T_{i \text { mean }}$ with the critical $u^{2}$ level is however much more pronounced compared to the change

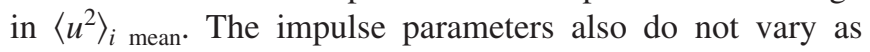
much as the number of impulses. Impulse intensity $\left(\delta=I_{i \text { std }} / I_{i \text { mean }}\right)$ increases in a linear fashion as the critical level moves down, mostly due to the decrease in the standard deviation of impulse. As the critical $u^{2}$ level approaches the average of $u^{2}$, the impulse intensity increases. Together with our earlier findings (shown in Fig. 7), this observation suggests that the distribution of impulse becomes more skewed for relatively lower critical $u^{2}$ levels. In the case of higher critical $u^{2}$ levels, however, the smaller peaks are not detected and the distribution of impulse, including only those extreme events with high impulse magnitudes, will approach a normal distribution.

It is apparent that the number of impulse events (those where $u^{2} \geq u_{\mathrm{cr}}^{2}$ ) is sensitive to $u_{\mathrm{cr}}^{2}$, but it is also clear that not all impulse events yield grain dislodgement. In the next section we investigate the relationship between those events that are above $u_{\mathrm{cr}}^{2}$ and critical impulse. ${ }^{25}$

\section{Critical impulse and number of grain entrainments}

Figures 9 and 10 imply that the turbulent channel flow contains flow structures with a potential to apply a range of impulse values varying over an order of magnitude. A threshold impulse value under the given bed and particle condi- tions can be defined by identifying an impulse level above which particle entrainment takes place. ${ }^{25}$ This constant impulse is approximated by a value of $\left\langle u^{2}\right\rangle_{i} T_{i}$ in the overlap region where both grain movement and no movement are observed on the plot of impulse, $\left\langle u^{2}\right\rangle_{i} T_{i}$, with respect to $\left\langle u^{2}\right\rangle_{i}$ (Fig. 12). This region lies between the "impulse" values of 0.0034 and $0.0095 \mathrm{~m}^{2} / \mathrm{s}$.

The computed critical impulse level is obtained from the average over this region as shown in Fig. 12 (data from E1-E8 are included). The obtained $\left\langle u^{2}\right\rangle_{i} T_{i}$ value approximates the threshold of impulse $\left(I_{\mathrm{cr}}\right)$, considering that all of the runs were performed under a single bed configuration and thus under the same threshold of movement condition. The uncertainty in detecting the critical impulse level in our analysis is attributed to the variations in the instantaneous drag coefficient. ${ }^{28}$

In order to investigate the role of the critical impulse level, the previous analysis using artificial critical $u^{2}$ levels (Sec. IV B) was extended. That is, a corresponding critical impulse level for each critical $u^{2}$ level (Table IV) was estimated as follows. The density of the particle corresponding to each of the critical $u^{2}$ levels given in Table IV was determined using Eq. (4). Note that here the size of the particle

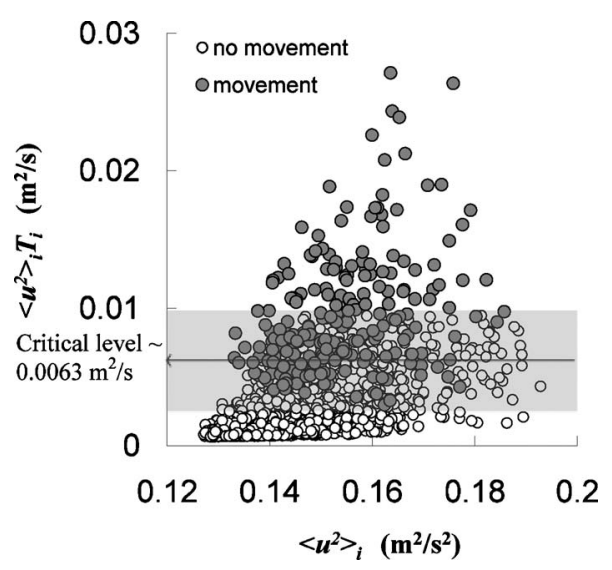

FIG. 12. $I_{i} \sim\left\langle u^{2}\right\rangle_{i} T_{i}$ vs $\left\langle u^{2}\right\rangle_{i}$ plot. The region where movement and no movement areas overlap is shown with a gray band between the impulse values of 0.0034 and $0.0095 \mathrm{~m}^{2} / \mathrm{s}$. Horizontal arrow indicates the critical impulse level. 


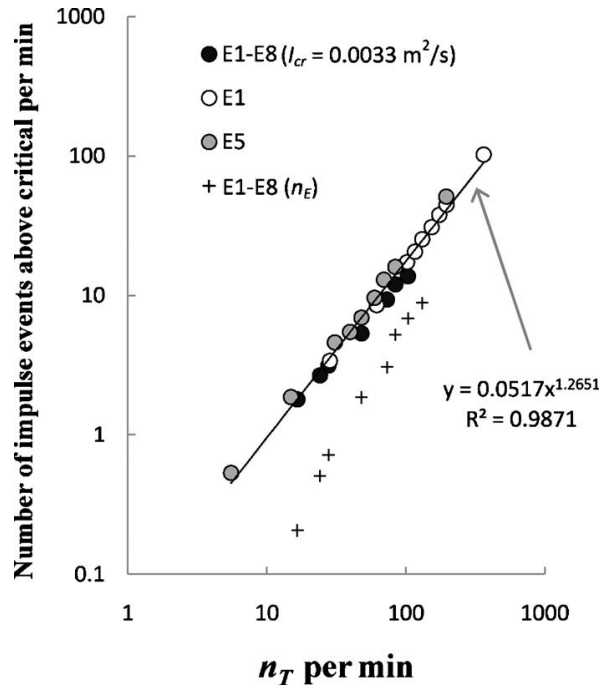

FIG. 13. The plot of number of impulse events above critical impulse per min vs total number of impulse events above critical $u^{2}$ per min. Data points with black circles are from all eight runs where a constant $I_{\text {cr }}$ was used. White and gray circles indicate results from runs E1 and E5, respectively, where various $I_{\mathrm{cr}}$ values were used. Data with the plus sign indicate the actual particle movements $\left(n_{E}\right)$ vs $n_{T}$ observed in each run.

remains the same. A theoretical initial velocity, $V_{\text {init }}$, of the particle with the calculated density, as a result of impulse, in the streamwise direction was estimated from the potential energy that the particle gains when it is elevated by an amount of $\Delta z(0.4 \mathrm{~mm})$ normal to the flow direction as it reaches the retaining pin, that is $V_{\text {init }}=\sqrt{2 g \Delta z}$. For simplicity the energy losses due to friction were ignored for this estimate. Using the change in the linear momentum of the particle, the impulse required (the critical level) was determined for each calculated particle density using $V_{\text {init }}$ and Eqs. (1), (3), and (4). The assumption here is that the impulse determined for each particle density represents a minimum (critical) impulse level that causes a detectible particle movement. The critical impulse level $\left(I_{\mathrm{cr}}\right)$ calculated in this fashion for the original particle density was $0.0033 \mathrm{~m}^{2} / \mathrm{s}$. We note that this value is near the lower impulse threshold detected using the actual observations of particle movement (Fig. 12).

Using the estimated artificial critical impulse levels, the data for each case in Table IV were reanalyzed to identify for each critical $u^{2}$ value the corresponding number of impulse events above the approximate critical impulse level. The analysis was performed using data from runs E1 and E5. Additionally, the number of impulse events above the critical impulse level of $0.0033 \mathrm{~m}^{2} / \mathrm{s}$ was also determined for all eight runs. The results are presented in Fig. 13 where the number of impulse events above critical $\left(I_{i} \geq I_{\mathrm{cr}}\right)$ per minute are plotted with respect to the total number of impulse events with $u^{2} \geq u_{\text {cr }}^{2}$ per minute, for all critical $u^{2}$ values tested using the data from runs E1 and E5. In addition, the number of actual particle movements $\left(n_{E}\right)$ observed in each of the eight runs was also plotted in the figure against the original $n_{T}$ (Table IV). There is clearly a strong relationship between the number of events above critical impulse and the total number of impulse events, $n_{T}$, regardless of the approach used to detect them, provided that the theoretical $I_{\text {cr }}$ is used. The

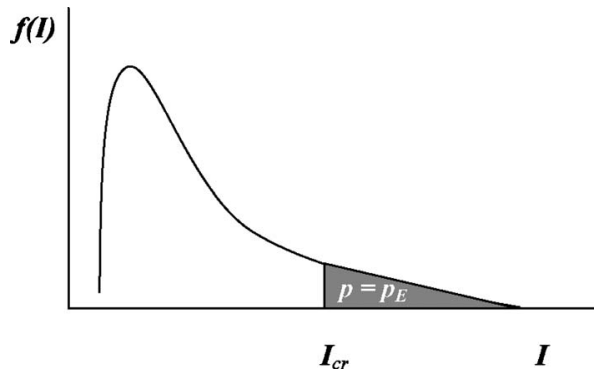

FIG. 14. Illustration of the probability analysis. The probability that a flow event will generate a level of impulse that exceeds a specified critical level, $I_{\text {cr }}$, is indicated by the shaded area and is assumed to be equal to the probability of particle entrainment, $p_{E}$.

functional relationship between the two variables and the coefficient of determination are given in Fig. 13 as an inset. This conformity indicates that the significant increase in both number of impulse events and the number of events above critical impulse with minute changes in either flow parameters or grain density is not a coincidence but a phenomenon inherent to turbulent flow-particle interactions. However, the actual number of ball movements is found to be lower than the number of events above a critical impulse with a potential to move the ball (black circles and plus signs in Fig. 13 are compared). One obvious reason for this difference is the fact that the calculated critical impulse used here is near the lower observed threshold for particle movement as mentioned earlier (Fig. 12). The next section examines the probability of particle movement taking into consideration the critical impulse values obtained here.

\section{Probability of particle entrainment}

Probability of particle entrainment has received much attention in the literature. ${ }^{9,17,18,36}$ It is commonly argued that the probability of exceeding of a given threshold level is equal to the probability of particle entrainment. ${ }^{18}$ Here we hypothesize that the probability, $p$, of occurrence of flow events that exceed the impulse threshold (Fig. 12) is equal to the probability of particle entrainment, $p_{E}$ (this concept is illustrated in Fig. 14). The former can be evaluated directly using Eq. (5) with the critical impulse value (in the form of $\hat{I}_{\mathrm{cr}}=I_{\mathrm{cr}} / I_{i}$ mean $)$. To examine the validity of this hypothesis the data for each of the series, E1-E8, were analyzed using three different critical impulse levels obtained from Fig. 12: the upper, lower, and average values associated with the overlap region in the figure.

We approximate the probability $p_{E}$ by the relative frequency of impulse events that yield particle dislodgement, i.e.,

$$
p_{E} \approx \frac{n_{E}}{n_{T}},
$$

$p$ and $p_{E}$ values obtained in this fashion are compared in Fig. 15. A good agreement is achieved between the two probability values, especially for higher entrainment rates when the critical impulse is taken as $0.0063 \mathrm{~m}^{2} / \mathrm{s}$ (Fig. 12). For comparison, $p$ values obtained using the upper and lower limits 


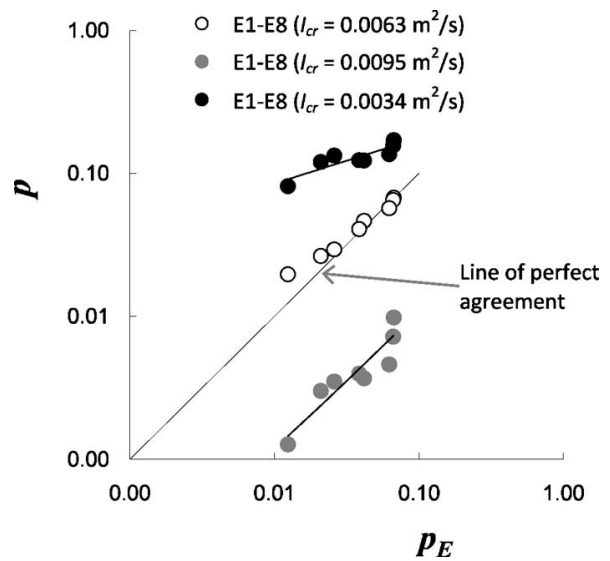

FIG. 15. Probability of particle entrainment vs probability of exceedance of critical impulse.

of the impulse threshold (Fig. 12) are also presented with respect to $p_{E}$ for all runs to reflect the effect on $p$ of the uncertainty in the critical impulse. As shown in Fig. 15, the variation of $p$ with $I_{\text {cr }}$ is significant. Nevertheless, the good agreement between $p$ and $p_{E}$, using $I_{\mathrm{cr}}=0.0063 \mathrm{~m}^{2} / \mathrm{s}$ confirms that the pdf given by Eq. (5) describes the data well and suggests that, despite the significant variability in particle entrainment frequency, consistent probability values for particle entrainment are obtained near threshold conditions when the frequency of impulse (i.e., $n_{T}$ ) is taken into account.

\section{IMPLICATIONS OF THE IMPULSE CONCEPT FOR LOW MOBILITY CONDITIONS}

Bedload measurements constitute an important component in the development of reliable formulae relating the amount of transported bed material to the flow parameters. Predictions of the limiting case of nearly zero bedload transport, the threshold of motion or critical condition, are even more challenging. The complexities at very low mobility conditions are associated with flow turbulence and bed microtopography parameters, the former being most prominent. In addition, the paucity of dependable data obtained under low shear stress and low bed material movement conditions hinders understanding of sediment transport processes at incipient conditions. Given these facts, Paintal's ${ }^{10}$ well known observation still remains to be explained.

We have shown in this study that for near threshold conditions, the particle entrainment rate increases significantly due to impulse events with only minute increases in the flow strength, interpreted in terms of boundary shear stress or similar parameters. The Shields stress values for instance in our tests varied between 0.008 and 0.011 , a change accompanied by nearly two orders of magnitude increase in the number of full particle dislodgements. This behavior exhibits strong qualitative similarities with the observations of Paintal ${ }^{10}$ and Helland-Hansen et al. ${ }^{12}$ Figure 16 shows particle movement at low Shields stresses using, as a surrogate measure, the number of impulses per minute above a critical impulse obtained from our experiments (see Sec. IV C). Dimensionless bed load parameter, $q^{*}$, reported by Paintal ${ }^{10}$

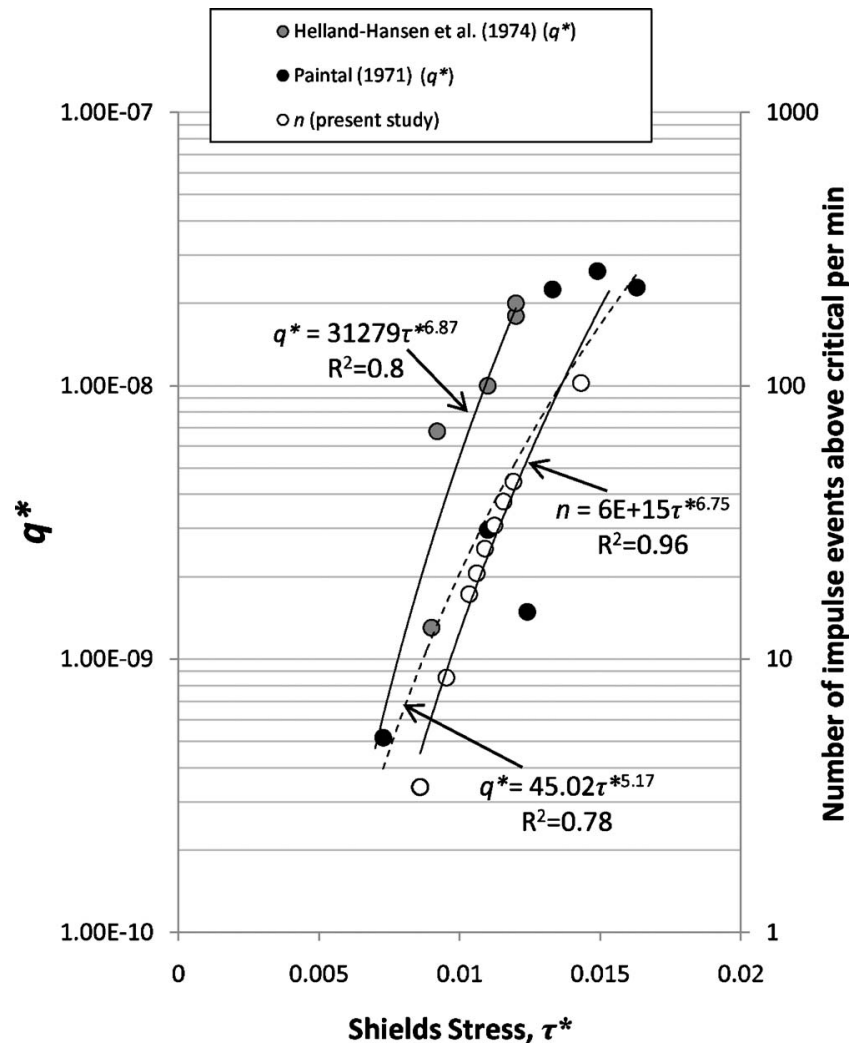

FIG. 16. Dimensionless bed load parameter vs shields stress (left $y$-axis) from Refs. 10 and 12. Note that data only in the $\tau^{*}$ range between 0.005 and 0.016 were used. The number of impulse events above critical impulse per min (right $y$-axis) vs shields stress is also plotted.

and Helland-Hansen et al. ${ }^{12}$ are included in the same figure for comparison purposes. Despite the variability in flow and bed material parameters employed by Paintal ${ }^{10}$ and HellandHansen et al., ${ }^{12}$ Fig. 16 shows a nearly two-orders-ofmagnitude increase in the number of impulses (with a potential to move the grains) per minute and in the dimensionless bed load values over a low Shields stress range. The observed similarity in the trends (see the exponents shown in the inset of Fig. 16) helps explain the well known, yet poorly understood, phenomenon observed first by Paintal. ${ }^{10}$ It also contributes to the validation of the impulse concept presented here for sediment entrainment at low shear conditions.

It is well understood now that the mean bed shear stress other than being a good descriptor of the mean forces cannot explain the local processes and resulting sediment movement at low shear stress conditions. The magnitude of the local hydrodynamic forces and their distributions form the basis of many stochastic incipient motion models to overcome the complexities of fluid-mobile boundary interactions (i.e., $u^{2} \geq u_{\mathrm{cr}}^{2}$ ). Our results on the other hand clearly show that impulse has far greater dynamical significance in terms of initial sediment movement.

This is clearly seen that the impulse events above critical $\left(I_{i} \geq I_{\mathrm{cr}}\right)$, rather than the peak forces (with $u^{2} \geq u_{\mathrm{cr}}^{2}$ ), are responsible for particle dislodgement (Fig. 12). Within the given shear stress range, a variability that is in close qualitative agreement with the findings of Paintal ${ }^{10}$ and HellandHansen et al. ${ }^{12}$ is observed in the number of impulse events 
above critical $\left(I_{i} \geq I_{\mathrm{cr}}\right)$ and not the number of peak forces for which $u^{2} \geq u_{\mathrm{cr}}^{2}$ (see Figs. 13 and 16). We believe therefore that the impulse concept presented here represents a significant step toward establishing incipient motion models with better predictive abilities.

Because the impulse is the germane criterion for describing the dislodgement of fully exposed as well as fully hidden particle configurations, ${ }^{25}$ it is expected to remain valid for the more general cases of variable local bed topography. Furthermore, determination of a critical impulse level depends on local bed conditions. In natural settings, grain size, shape, exposure and packing density vary greatly in a probabilistic manner ${ }^{8}$ making also the resistance (critical impulse) of grains to vary spatially. Therefore the threshold of real sediment movement in both space and time requires consideration of both the impulse potential of the turbulent flow and the distribution of critical impulse. Nevertheless, future work will be necessary to demonstrate this result conclusively.

\section{CONCLUSIONS}

We showed for a range of flow conditions and particle entrainment rates that the impulse is a better suited parameter to describe the role of turbulence fluctuations on the particle movement for the given bed configuration and under incipient conditions. Using a particle tracking technique that offers high temporal and spatial resolution in detecting the motion of a single test particle, we found that the particle entrainment rate is extraordinarily sensitive to minute changes in the gross flow parameters. This finding is in agreement with well known observations of others available in the literature.

A log-normal distribution was found to describe the impulse data well (Figs. 8 and 9). Equation (5), for the lognormal distribution, is a function of impulse intensity, $\delta$, alone, which can be determined experimentally or using Eq. (6) for the given $\mathrm{Re}^{*}$ range. Equation (6) is therefore useful for further studies under similar bed and low shear stress configurations.

We demonstrated that the impulse imparted by the turbulent stream on the mobile grain has to be sufficiently large in order to cause dislodgement. Combinations of force duration and magnitude that entrained the particle were observed to be in general above a critical impulse value (Fig. 12). The durations of applied force varied an order of magnitude for corresponding relatively small range of the force ( $u^{2}$ values). The resulting strong dependency of impulse values on the duration of applied forcing suggests that the magnitude of drag force is necessary but not sufficient to characterize the threshold of motion.

We also investigated the role of $u_{\mathrm{cr}}^{2}$ (representing the minimum drag force necessary to entrain the particle) on the number of detected impulses. Additionally, the role of critical impulse level on the number of impulse events with a potential to move the grain was examined. The results showed that minute changes in the $u_{\mathrm{cr}}^{2}$ and the critical impulse leads to significant variations in the number of total impulse events and events with a potential to dislodge the grain, respectively. This analysis substantiates and helps explain the ob- served nearly two-orders-of-magnitude increase in the particle movement rate with $35 \%$ increase in the bed shear stress.

The derived pdf [Eq. (5)] of dimensionless impulse was used together with an approximated critical impulse level to find the probability of particle entrainment. Probabilities obtained in this fashion were checked against experimentally determined particle entrainment probabilities and a good agreement was achieved when an approximated critical impulse level was used.

Finally, the significant increase in the number of impulse events above critical impulse with small increases in the bed shear stress was found to be qualitatively similar to the significant increase in the bed load transport observed by others under comparable low mobility conditions.

Our results using the impulse concept link the characteristics of turbulent flow to particle entrainment and, despite the simplified bed geometry employed, have far reaching consequences in describing the inadequacies of existing incipient motion models, as well as improving our ability to model a wide range of river mechanics and other geomorphology related phenomena.

\section{ACKNOWLEDGMENTS}

The support of the National Science Foundation (Grant Nos. EAR-0439663 and EAR-0738759) and Army Research Office for this study is gratefully acknowledged.

\section{APPENDIX: DERIVATION OF THE PDF FOR IMPULSE}

The log-normal distribution for a random variable $x$ is defined by

$$
f(x)=\frac{1}{\sigma \sqrt{2 \pi} x} \exp \left[-\frac{1}{2}\left(\frac{\ln (x)-\mu}{\sigma}\right)^{2}\right],
$$

where $\mu$ and $\sigma$ are the mean and standard deviation of the variable's natural logarithm, $\ln (x) . x_{\text {mean }}$ and $x_{\text {std }}$ representing the mean and standard deviation of the variable $x$, and

$$
c_{v}=\frac{x_{\text {std }}}{x_{\text {mean }}},
$$

where $c_{v}$ is the coefficient of variation of $x, \mu$, and $\sigma$, can be defined as

$$
\mu=\ln \left(\frac{x_{\text {mean }}}{\sqrt{1+\left(\frac{x_{\text {std }}}{x_{\text {mean }}}\right)^{2}}}\right)=\ln \left(\frac{x_{\text {mean }}}{\sqrt{1+c_{v}^{2}}}\right),
$$

and

$$
\sigma=\sqrt{\ln \left[1+\left(\frac{x_{\text {std }}}{x_{\text {mean }}}\right)^{2}\right]}=\sqrt{\ln \left(1+c_{v}^{2}\right)},
$$

respectively. ${ }^{37}$ Equation (A1), using Eqs. (A2)-(A4), can be rewritten as 


$$
\begin{aligned}
f(x)= & \frac{1}{\sqrt{2 \pi \ln \left(1+c_{v}^{2}\right) x}} \\
& \times \exp \left[-\frac{\left(\ln \left(\frac{x}{x_{\text {mean }}}\right)+\ln \sqrt{1+c_{v}^{2}}\right)^{2}}{2 \ln \left(1+c_{v}^{2}\right)}\right] .
\end{aligned}
$$

The pdf given by Eq. (A5) can be transformed ${ }^{37}$ for a new dimensionless random variable $\hat{x}$, which is defined as

$$
\hat{x}=\frac{x}{x_{\text {mean }}} .
$$

The pdf transformation yields

$$
f(\hat{x})=\frac{1}{\sqrt{2 \pi \ln \left(1+c_{v}^{2}\right)} \hat{x}} \exp \left[-\frac{\left(\ln \hat{x}+\ln \sqrt{1+c_{v}^{2}}\right)^{2}}{2 \ln \left(1+c_{v}^{2}\right)}\right] .
$$

Equation (A7) is dependent only on $c_{v}$. Here $x$ is the impulse, $I=\left\langle u^{2}\right\rangle T$, and $c_{v}$ is the impulse intensity, $\delta$, given by

$$
c_{v}=\delta=\frac{I_{\text {std }}}{I_{\text {mean }}} .
$$

If the impulse is nondimensionalized as follows:

$$
\hat{x}=\hat{I}=\frac{I}{I_{\text {mean }}},
$$

Eqs. (A7)-(A9) then yield

$$
f(\hat{I})=\frac{1}{\sqrt{2 \pi \ln \left(1+\delta^{2}\right)} \hat{I}} \exp \left[-\frac{\left(\ln \hat{I}+\ln \sqrt{1+\delta^{2}}\right)^{2}}{2 \ln \left(1+\delta^{2}\right)}\right] .
$$

Note that $\delta=\delta_{I}$, the latter being the intensity of the dimensionless impulse $\hat{I}$. Equation (A10) shown above is the pdf of dimensionless impulse, $\hat{I}$ as a function of $\delta$.

\footnotetext{
${ }^{1}$ A. Shields, 1936. "Anwendung der aehnlichkeitsmechanik und der turbulenzforschung auf die eschiebebewegung" Mitteilungen der Preussiischen versuchsanstalt fur wasserbau und schiffhau, Heft 26, Berlin (in German) [English translation by W. P. Ott and J. C. van Uchelen, Publication No. 167, Hydrodynamics Lab., California Inst. of Technology, Pasadena, CA, 1950].

${ }^{2}$ J. M. Buffington and D. R. Montgomery, "A systematic analysis of eight decades of incipient motion studies, with special reference to gravelbedded rivers," Water Resour. Res. 33, 1993 (1997).

${ }^{3}$ A. A. Kalinske, "Movement of sediment as bed load in rivers," Trans., Am. Geophys. Union 28, 615 (1947).

${ }^{4}$ A. J. Sutherland, "Proposed mechanism for sediment entrainment by turbulent flows," J. Geophys. Res. 72, 6183, doi:10.1029/JZ072i024p06183 (1967).

${ }^{5}$ A. D. Heathershaw and P. D. Thorne, "Sea-bed noises reveal role of turbulent bursting phenomenon in sediment transport by tidal currents," Nature (London) 316, 339 (1985).

${ }^{6}$ J. Nelson, R. L. Shreve, S. R. McLean, and T. G. Drake, "Role of near-bed turbulence structure in bed-load transport and bed-form mechanics," Water Resour. Res. 31, 2071, doi:10.1029/95WR00976 (1995).

${ }^{7}$ B. M. Sumer, L. H. C. Chua, N. S. Cheng, and J. Fredsoe, "Influence of turbulence on bed load sediment transport," J. Hydrol. Eng. 129, 585 (2003).

${ }^{8}$ J. W. Kirchner, W. E. Dietrich, F. Iseya, and H. Ikeda, "The variability of critical shear stress, friction angle, and grain protrusion in water-worked sediments," Sedimentology 37, 647 (1990).
}

${ }^{9}$ A. J. Grass, in The Influence of Boundary Layer Turbulence on the Mechanics of Sediment Transport, edited by B. M. Sumer and A. Muller (Balkema, Rotterdam, 1983), pp. 3-17.

${ }^{10}$ A. S. Paintal, "Concept of critical shear stress in loose boundary open channels," J. Hydr. Div. 9, 91 (1971).

${ }^{11}$ J. D. Fenton and J. E. Abbot, "Initial movement of grains on a stream bed: The effect of relative protrusion," Proc. R. Soc. London 352A, 523 (1977).

${ }^{12}$ E. Helland-Hansen, P. C. Klingeman, and R. T. Milhous, "Sediment transport at low Shields-paramter values," J. Hydr. Div. 100, 261 (1974).

${ }^{13}$ B. Hofland, "Rock \& Roll: Turbulence-induced damage to granular bed protections," Ph.D. thesis, Department of Civil Engineering and Geosciences, Delft University of Technology, The Netherlands (2005).

${ }^{14}$ H. A. Einstein and E. A. El-Samni, "Hydrodynamic forces on a rough wall," Rev. Mod. Phys. 21, 520 (1949).

${ }^{15}$ A. J. Grass, "Initial instability of fine bed sand," J. Hydr. Div. 96, 619 (1970).

${ }^{16}$ I. McEwan and J. Heald, "Discrete particle modeling of entrainment from flat uniformly sized sediment beds," J. Hydrol. Eng. 127, 588 (2001).

${ }^{17}$ A. B. Shvidchenko and G. Pender, "Flume study of the effect of relative depth on the incipient motion of coarse uniform sediments," Water Resour. Res. 36, 619, doi:10.1029/1999WR900312 (2000).

${ }^{18}$ A. N. Papanicolaou, P. Diplas, N. Evaggelopoulos, and S. Fotopoulos, "Stochastic incipient motion criterion for spheres under various bed packing conditions," J. Hydrol. Eng. 128, 369 (2002).

${ }^{19}$ N. S. Cheng and Y. M. Chiew, "Pick-up probability for sediment entrainment," J. Hydrol. Eng. 124, 232 (1998).

${ }^{20}$ M. W. Schmeeckle and J. M. Nelson, "Direct numerical simulation of bedload transport using a local dynamic boundary condition," Sedimentology 50, 279 (2003).

${ }^{21}$ F. C. Wu and Y. J. Chou, "Rolling and lifting probabilities for sediment entrainment," J. Hydrol. Eng. 129, 110 (2003).

${ }^{22}$ S. Vollmer and M. G. Kleinhans, "Predicting incipient motion, including the effect of turbulent pressure fluctuations in the bed," Water Resour. Res. 43, W05410, doi:10.1029/2006WR004919 (2007).

${ }^{23}$ M. S. Yalin, River Mechanics (Pergamon, Oxford, 1992).

${ }^{24} \mathrm{M}$. Balakrishnan, "The role of turbulence on the entrainment of a single sphere and the effects of roughness on fluid-solid interaction," Ph.D. dissertation, Virginia Polytechnic Institute and State University, Blacksburg, VA (1997).

${ }^{25}$ P. Diplas, C. L. Dancey, A. O. Celik, M. Valyrakis, K. Greer, and T. Akar, "The role of impulse on the initiation of particle movement under turbulent flow conditions," Science 322, 717 (2008).

${ }^{26}$ J. Gessler, in River Mechanics, edited by H. W. Shen (Water Resources, Forth Collins, 1971), Chap. 7.

${ }^{27} \mathrm{C}$. R. White, "The equilibrium of grains on the bed of a stream," Proc. R. Soc. London, Ser. A 174, 322 (1940).

${ }^{28}$ M. W. Schmeeckle, J. M. Nelson, and R. L. Shreve, "Forces on stationary particles in near-bed turbulent flows," J. Geophys. Res. 112, F02003, doi:10.1029/2006JF000536 (2007).

${ }^{29}$ J. S. Bridge and S. J. Bennett, "A model for the entrainment and transport of sediment grains of mixed sizes, shapes, and densities," Water Resour. Res. 28, 337, doi:10.1029/91WR02570 (1992).

${ }^{30} \mathrm{~B}$. Hofland and J. Battjes, "Probability density function of instantaneous drag forces and shear stresses on a bed," J. Hydrol. Eng. 132, 1169 (2006).

${ }^{31}$ T. Song, W. H. Graf, and U. Lemmin, "Uniform flow in open channels with movable gravel bed," J. Hydraul. Res. 32, 861 (1994).

${ }^{32}$ N. S. Cheng and A. W. K. Law, "Fluctuations of turbulent bed shear stress," J. Eng. Mech. 129, 126 (2003).

${ }^{33}$ B. S. Wu, A. Molinas, and P. Y. Julien, "Bed-material load computations for nonuniform sediments," J. Hydraul. Eng. 130, 1002 (2004).

${ }^{34} \mathrm{H}$. Mouri, A. Hori, and M. Takaoka, "Large-scale lognormal fluctuations in turbulence velocity fields," Phys. Fluids 21, 065107 (2009).

${ }^{35}$ E. Limpert, W. A. Stahel, and M. Abbt, "Log-normal distributions across the sciences: Keys and clues," Bioscience 51, 341 (2001).

${ }^{36}$ C. L. Dancey, P. Diplas, A. Papanicolaou, and M. Bala, "Probability of individual grain movement and threshold condition," J. Hydraul. Eng. 128, 1069 (2002).

${ }^{37}$ E. J. Gumbel, Statistics of Extremes (Columbia University Press, New York, 1958). 